\title{
Perception of duration in the parvocellular system
}

\section{Guido M. Cicchini*}

Visual Psychophysics, Institute of Neuroscience, Consiglio Nazionale delle Ricerche, Pisa, Italy

\section{Edited by:}

Warren H. Meck, Duke University, USA

\section{Reviewed by:}

Virginie Van Wassenhove, Cognitive Neuroimaging Unit, France

Alan Johnston, University College London, UK

\section{*Correspondence}

Guido M. Cicchini, Visual Psychophysics, Institute of Neuroscience, Consiglio Nazionale delle Ricerche, Via Moruzzi.

1 - 56124 Pisa, Italy.

e-mail: cicchini@in.cnr.it
Both theoretical and experimental evidence suggests that duration perception is mediated preferentially by the color-blind but high temporally sensitive luminance pathway. In this experiment we tested whether color modulated stimuli and high spatial frequency luminance modulated stimuli, which are known to be relayed mostly by the slow parvocellular system, are able to elicit reliable sense of duration. We show that ramped color modulated stimuli seem to last less than luminance modulated stimuli matched for visibility. The effect is large, about $200 \mathrm{~ms}$ and is constant at all durations tested (range 500-1100 ms). However, high spatial frequency luminance stimuli obtain duration matches similar to those of low spatial frequency luminance modulated stimuli. The results at various levels of contrast and temporal smoothing indicate that equiluminant stimuli have higher contrast thresholds to activate the mechanisms which time visual stimuli. Overall the results imply that both the magnocellular and the parvocellular systems access reliably the timing mechanisms with a difference only in the way these are engaged.

\section{INTRODUCTION}

Despite subjective time is a perceptual dimension that transcends each sensory modality, recent evidence is showing that the estimation of brief temporal intervals is computed by multiple parallel temporal units deeply rooted in sensory processes (Buonomano and Merzenich, 1995; Ivry and Spencer, 2004; Mauk and Buonomano, 2004; Johnston et al., 2006; Johnston, 2010). Psychophysical results indicate that one first division for temporal processing regards events the modality which signals the event. Intervals in different modalities are processed with different sensitivities (Goodfellow, 1934; Grondin, 1993; Burr et al., 2009; Cicchini et al., 2012), undergo selective distortions (Yarrow et al., 2001; Morrone et al., 2005, 2010; Johnston et al., 2006; Binda et al., 2009), and can be calibrated one with the other (Fujisaki et al., 2004; Miyazaki et al., 2006; Stetson et al., 2006; van Wassenhove et al., 2008). In addition it is now becoming clear that even within the same modality multiple clocks exist. For instance, in vision adaptation to drifting gratings distorts events only in the adapted location (Johnston et al., 2006; Ayhan etal., 2009); similarly, if an interval is defined by two flashes in the same location, it is estimated correctly even when attention is draw away (Cicchini and Morrone, 2009). Not least perceived timing depends on very basic stimulus features (such as speed, contrast, size), indicating that the network for temporal processing extends to and is in partial overlap with purely sensory processes (Roelofs and Zeeman, 1951; Brown, 1995; Terao et al., 2008; Cicchini and Morrone, 2009). All of these notions go hand in hand with the concept that even small neuronal ensembles, not specialized for temporal processing, are able to encode the passage of time (Buonomano and Merzenich, 1995; Yamazaki and Tanaka, 2005; Karmarkar and Buonomano, 2007).

Within vision a further parcellation seems to occur. Among the multiple parallel visual channels, those that support temporal processing should be the ones best suited for visual transients.
Consistently moving and flickering stimuli are perceived as lasting longer than static stimuli (Roelofs and Zeeman, 1951; Brown, 1995; Kanai et al., 2006; Kaneko and Murakami, 2009) and adaptation to high speed gratings induces a decrease of duration (Johnston et al., 2006; Burr et al., 2007; Ayhan et al., 2009).

The demand for fast and reliable temporal information about the environment seems to be met at retino-cortical level by the existence of the magnocellular visual pathway. Neurons along the magnocellular pathway have relatively large receptive fields and fast response properties (Livingstone and Hubel, 1988). These cells have also diphasic impulse response functions which are well suited to capture changes in visual stimuli and relay temporal information with good temporal resolution. The spatial frequency selectivity of magnocellular neurons is bandpass with a peak just below 1 cpd and a cut-off spatial frequency that ranges between 2 and 6 cpd depending on the temporal frequency of the stimulus (Derrington and Lennie, 1984) and its eccentricity (Alitto et al., 2011). Indeed, the selectivity for low spatial frequencies and high temporal frequencies suggests that the magnocellular information is crucial to perceive motion and rapid changing events (Livingstone and Hubel, 1988; Levitt et al., 2001; Leonova et al., 2003).

The other main subcortical pathway, the parvocellular one, seems much less suited for temporal processing with slower peak responses and low pass temporal characteristics. In agreement with physiology reaction times and VEP, responses to luminance modulated stimuli are faster than responses to equiluminant stimuli equated for contrast (Burr et al., 1998; Burr and Corsale, 2001; McKeefry et al., 2003). In terms of spatial selectivity neurons in the parvocellular pathway extend well beyond the frequencies of the magnocellular system and respond to up to $15-20 \mathrm{cpd}$ for color and luminance defined gratings with a peak of sensitivity at around $10 \mathrm{cpd}$. Parvocellular neurons afferents are the most common input to visual cortex making up to $80 \%$ of visual afferent signals 
and their putative role is to support finer aspects of visual analysis for tasks that are not demanding of high temporal resolution (Ingling Jr. and Martinez-Uriegas, 1983; Kaplan and Benardete, 2001; Gegenfurtner and Kiper, 2003).

All these lines of evidence indicate that magnocellular transmission has a primary role in mediating the fine temporal aspects of visual processing including onsets and offsets and when magnocellular transmission is impaired (like after adaptation or in dyslexia) temporal compression is expected (Johnston et al., 2006, 2008; Johnston, 2010; Ayhan et al., 2011).

In this study I tried to extend this notion by investigating perceived duration of stimuli relayed by the parvocellular pathway. I found that equiluminant stimuli are perceived as much shorter than luminance modulated stimuli with a constant difference at all durations in the range 500-1100 ms. Further experiments estimated the visibility threshold at which timing starts and stops and confirm that equiluminant and luminance modulated stimuli differ only in the way they engage timing mechanism but they share the mechanism which integrates the passage of time.

These results have been published in abstract form (Cicchini and Tomassini, 2008).

\section{MATERIALS AND METHODS PARTICIPANTS}

Overall nine right-handed participants aged 20-32 (two men, seven women) took part in these experiments. All had normal or corrected-to-normal vision, and had no reported hearing deficits. The experimental procedures were approved by the Ethics Committee of Università Vita-Salute San Raffaele. All the subjects but the author were naive to the purpose of the experiment and gave informed consent to participate to the study.

\section{APPARATUS}

Subjects sat 57 or $228 \mathrm{~cm}$ away from a calibrated Silicon Graphics 21" CRT Trinitron monitor running at a resolution of $800 \times 600$ pixels at a frame rate of $100 \mathrm{~Hz}$. Stimuli were generated through a VSG 2/5 Graphics Card (Cambridge Research Systems) controlled via Matlab software and the VSG routines. The red phosphor had CIE coordinates of $x=0.56, y=0.34$, while the green phosphor had coordinates $x=0.3$ and $y=0.55$. Experiments were run in a dimly lit room.

\section{EQUILUMINANCE}

For each subject equiluminance point was determined via standard flicker photometry reversing at $15 \mathrm{~Hz}$ a $1 \mathrm{cpd}$ Gabor patch with the maximum possible red gun modulation and a variable green to red luminance ratio. The subject adjusted the amplitude of the green color component until the percept of minimum flicker was obtained. The procedure was repeated five times and the green-red ratio yielding minimum flicker were averaged.

\section{STIMULI}

The stimuli for the time judgments were gratings windowed by a Gaussian of $3^{\circ}$ of standard deviation presented at fixation. Achromatic stimuli were obtained putting in phase the red and green profiles of the underlying sinusoid. Color modulation was obtained putting in antiphase the two calibrated sinusoids. Overall three types of stimuli were employed, Achromatic $1 \mathrm{cpd}$, Chromatic $1 \mathrm{cpd}$, Achromatic of $8 \mathrm{cpd}$. The first type of stimulus should excite preferentially the magnocellular system, the latter two the parvocellular system.

\section{TEMPORAL SMOOTHING}

Since perception of duration is altered by the presence of changes and transitions, care was taken to ensure that both luminance and colored patches had a smooth onset and offset. For this reason the square wave temporal profile of the stimuli with a given nominal duration was smoothed with a Gaussian temporal window. In most experiments the Gaussian window had a $\sigma$ of $100 \mathrm{~ms}$. In one experiment, which tested directly the effect of stimulus ramping, the smoothing constant assumed values between 50 and $180 \mathrm{~ms}$. Also with these shorter constants, the stimuli never yielded the percept of a brisk transient and duration matches were always made upon perceptually similar stimuli.

Throughout the paper stimuli are described in terms of their nominal duration (i.e., the duration of the underlying square wave). This quantity does not correspond necessarily to the time the stimulus exceeds detection threshold. A stimulus with a contrast barely above detection thresholds remains visible for less than the nominal duration. If the stimulus has a contrast of twice visibility thresholds, time above threshold equals nominal duration. At higher contrasts the time the stimulus is above threshold is longer than nominal duration (see Figure 3A). Indicatively at a contrast of 15 times the visibility threshold (which is a common choice throughout the work) a Gabor of nominal duration $1000 \mathrm{~ms}$ is above detection threshold for $1212 \mathrm{~ms}$.

\section{VISIBILITY THRESHOLDS}

Visibility thresholds were measured with a standard 2 IFC procedure where two 3-s blocks (one empty, one containing a target stimulus) were presented in random order and subjects had to indicate which of the two contained a target. The target stimulus lasted $600 \mathrm{~ms}$ and was smoothed in time with a $\sigma$ of $100 \mathrm{~ms}$. Contrast was varied by a QUEST routine and data were fitted by a psychometric function. The point of passage through the $75 \%$ correct responses was taken as the visibility threshold for that type of stimulus.

Visibility thresholds serve as a unit for standardizing stimuli across subjects. All contrasts used in the paper are expressed in terms of "visibility" which is "multiples of the detection contrast." Visibility as such is a normalized physical quantity and bears no implication on the perceived contrast and or salience of the stimulus.

\section{DURATION COMPARISON}

In the duration comparison subjects were asked to compare the duration of two smoothed Gabors presented sequentially at fixation. One of the two stimuli, the "reference," was an achromatic Gabor of $1 \mathrm{cpd}, 15$ times the visibility level and had a fixed duration throughout an experimental session. The other stimulus, the "test," had visibility, color and spatial frequency which changed with the experimental condition. The duration of the "test" varied on every trial following a QUEST routine (Watson and Pelli, 1983) curtailed at 200 and 3000 ms. Duration matches have been 
performed with several durations of the reference (from 500 to $1000 \mathrm{~ms}$ ) and for each, a separate psychometric function has been drawn.

Each trial begun with a variable foreperiod (from 1000 to $2500 \mathrm{~ms}$ ) in which only a small black fixation dot (1 pixel) was present at the center of a uniform yellow screen. Reference and test were then presented in random order separated by a pause of $1000-2500 \mathrm{~ms}$. At the end of the second stimulus the subjects indicated which of the two stimuli had been presented longer. Each experimental session comprised about 40 trials and, on average, 200 trials per condition were collected.

The point of subjective equality (PSE) of the reference and test stimulus duration was estimated from the median of the best fitting cumulative Gaussian psychometric function. This value corresponds to how long the test stimulus has to be presented for in order to equate the reference stimulus. If the test stimulus is underestimated the match is obtained at longer exposures and matching durations are higher. The precision (i.e., JND) in the duration task was calculated as the difference between the third and first quartiles divided by 2 .

Standard errors of PSE and JND were calculated via a bootstrap procedure which re-sampled with re-emission 500 times the set of stimulus-responses. The standard deviation of the re-sampled values yields an estimate of the standard error of the measurement (Efron and Tibshirani, 1994).

\section{AUDIOVISUAL TEMPORAL ORDER JUDGMENT}

In a separate experiment subjects were asked to compare the onset (or offset) of the test stimulus with respect to a brief auditory stimulus (a brief burst of white noise windowed by a Gaussian envelope of $5 \mathrm{~ms}$ standard deviation). In the case of the onset AVTOJ the pause preceding the stimulus ranged between 1000 and $2500 \mathrm{~ms}$. In the case of the temporal offset judgment the duration of the grating varied at random between 500 and $1200 \mathrm{~ms}$. The time between the auditory stimulus and the nominal beginning or end of the visual stimulus were selected by a QUEST routine. At least 60 trials were collected per condition and data were analyzed via fitting a cumulative Gaussian distribution and taking the median of the best fitting distribution.

\section{MODELING CONTRAST DEPENDENCE}

Duration matches for luminance modulated and equiluminant stimuli have been fitted with a simple clock model that is engaged when the stimulus attains a certain contrast. The clock's threshold is a free parameter and it has been assumed that the luminance and the color modulated stimuli may trigger timing with a separate threshold. Thus the whole dataset has been fitted with two free parameters.

The data fitting procedure analyzes first the achromatic dataset calculating first how much time the reference stimulus (15 times visibility) exceeds threshold and then how much time the test stimuli (of various contrasts) spend above threshold. The difference between the two values gives an estimate of how subjective duration should vary as function of contrast. The procedure is repeated for several candidate thresholds and the best fitting threshold for achromatic dataset is selected. The threshold for achromatic stimuli intrinsically provides also an estimate of how much time the reference stimulus ( $1 \mathrm{cpd}$ Achromatic, 15 times visibility) engages the clock.

Data fitting is then performed on equiluminant stimuli calculating how much time a clock is engaged as function of visibility and assuming that this value is compared to the recorded duration of the achromatic reference. Predictions for several thresholds for color stimuli are compared and the best fitting value is chosen.

\section{RESULTS}

\section{PERCEIVED DURATION IN THE MAGNOCELLULAR AND PARVOCELLULAR SYSTEMS}

Figure 1 shows the results when subjects had to compare the duration of a color modulated stimulus to that of a reference luminance modulated stimulus. Figures 1A,B present the data for two illustrative subjects at various reference durations (red symbols). In both subjects the data points lie above the diagonal indicating that subjects needed a longer presentation of the color modulated stimulus to match the duration of a reference luminance modulated stimulus. This corresponds to a temporal underestimation of the color modulated stimulus. Figure 1C shows results for four subjects at two representative durations (500 and $1000 \mathrm{~ms}$ ). On average the effect was $195 \mathrm{~ms}$ at $500 \mathrm{~ms}$ and $174 \mathrm{~ms}$ at $1000 \mathrm{~ms}$ $[F(3)=13.6, p<0.05]$ and was not proportional to the base interval duration $[F(3)=0.14, p>0.7]$.

Figures 1D-F report the precision of the temporal judgments as function of stimulus duration. JNDs increase with stimulus duration, complying with Weber's law for temporal judgments. On average color modulated stimuli yielded less precise judgments by $31 \mathrm{~ms}[F(3)=9.9, p=0.05]$. This effect however is similar at the two base durations $[28 \pm 8 \mathrm{~ms}$ at $500 \mathrm{~ms}, 33 \pm 8 \mathrm{~ms}$ at $1000 \mathrm{~ms}$, $F(3)=0.15, p>0.7]$ speaking against the idea that the increase of noise in the judgment is caused by the recruitment of a more noisy temporal integrator.

Interestingly the effect did not generalize to other stimuli relayed by the parvocellular pathway such as $8 \mathrm{cpd}$ achromatic Gabors. Figure 2 reports the results in a different subject pool which confirmed the effect with color modulated stimuli [ $185 \mathrm{~ms}$ across all durations, $t(4)=9.9$ and 14.7 for the two base durations, both $t$-test $p<0.01]$ but found a much weaker effect for $8 \mathrm{cpd}$ achromatic gratings ( $9 \mathrm{~ms}$ at $500 \mathrm{~ms}$ and $20 \mathrm{~ms}$ at $1000 \mathrm{~ms}$, both $t$-test $p>0.10)$.

This suggests that some stimuli relayed by the parvocellular pathway are able to engage effectively temporal duration mechanisms.

\section{CONTRAST THRESHOLD FOR TEMPORAL MARKERS}

All the effects encountered so far are independent of stimulus duration and seem to indicate that the differences in perceived duration of equiluminant stimuli arise in the process of engaging and disengaging the timing mechanisms. In the remainder of the paper I present three complementary experiments that estimated the contrasts thresholds at which timing starts and stops.

\section{Effect of stimulus visibility}

The first technique was to measure perceived duration as function of contrast and comparing perceptual data with the predictions of a simple threshold model. 


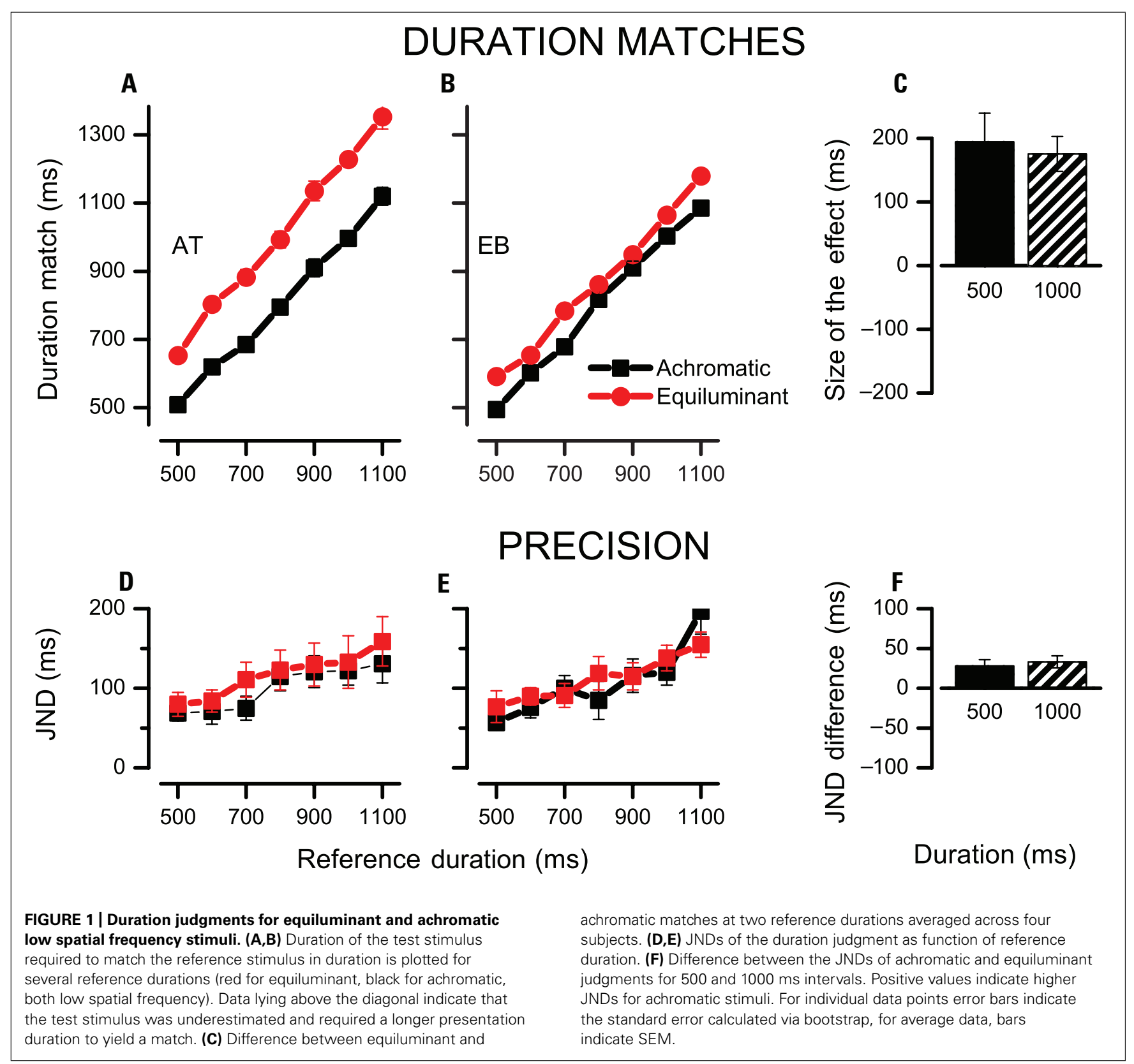

Figure 3A illustrates how the interval recorded by a clock varies as function of visibility and the clock threshold. High contrast stimuli pass more time above threshold than low contrast stimuli. Due to temporal smoothing, the modulation can be quite large, up to several hundred milliseconds especially at higher clock thresholds (see dashed lines).

Figures 3B,C data points show duration matches for achromatic and color modulated stimuli of $1 \mathrm{cpd}$ at various visibility levels (i.e., multiples of detection contrast). At lower contrasts both achromatic and color modulated stimuli require longer presentations indicating that lowering the contrast decreases perceived duration.

Figures 3B,C overlay on raw data the predictions of models which start timing at different visibility levels. Interestingly a simple model which starts timing at detection (gray curves) captures just coarsely the relationship between duration and visibility levels $\left(R^{2}=0.73\right.$ and 0.84$)$. Models which assume higher thresholds fare better $\left(R^{2}=0.98\right.$ and 0.99$)$. The thresholds which fit best the achromatic dataset assume that timing is engaged at 3.3 and 2.5 visibility contrast for the subjects GMC and OS respectively (black curves). Best fitting values for equiluminant stimuli are obtained assuming that clock thresholds are 7.3 and 8.2 times detection thresholds $\left(R^{2}=0.96\right.$ and 0.95$)$.

In the case of equiluminant stimuli the model captures well human performance only between moderate and high contrasts. This is because a threshold model predicts that when contrast approaches the clock threshold, a very dramatic decrease of perceived duration should be found. Indeed at contrasts 


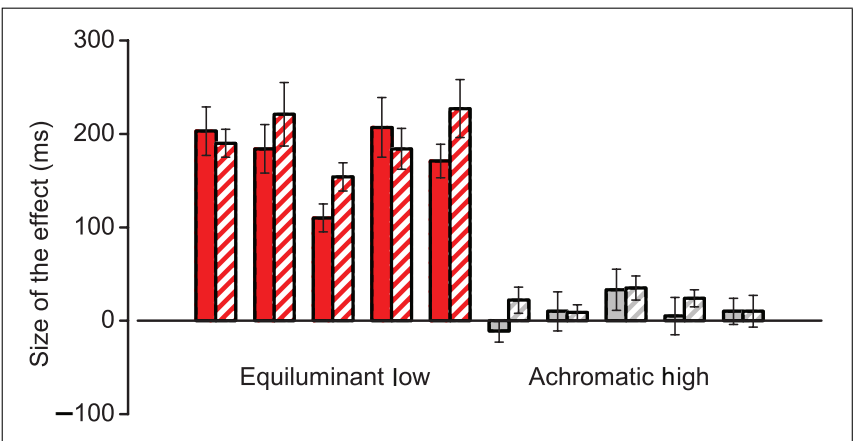

FIGURE 2 | Duration bias for stimuli relayed in the parvocellular pathway. Difference between test stimulus and reference stimulus matches for two types of stimuli relayed by the parvocellular pathway. Positive values indicate an underestimation of the test stimulus. Red bars are individual data for equiluminant low spatial frequency stimuli and gray bars for achromatic high spatial frequency stimuli. Solid and hatched bars refer to matches obtained with reference stimuli of 500 and $1000 \mathrm{~ms}$ respectively. Error bars indicate bootstrap standard errors.

lower than clock threshold, such a model predicts that subjects should not even be able to do the task. In these suboptimal conditions it is likely that alternative timing strategies may come into play.

It is also interesting to compare the data to a model which assumes that equiluminant and achromatic stimuli are timed with the same criteria (i.e., thresholds) and that all the difference relies on a fixed bias. Such fits can be obtained adding 220 and $280 \mathrm{~ms}$ to the luminance curves but provide just rough fits to the data ( $R^{2}=0.68$ and 0.63 for the two subjects).

\section{Effect of temporal smoothing}

The data presented so far indicate that the timing mechanisms may be triggered at different visibility levels depending on the type of stimulus. Here we try to reinforce this conclusion with a technique that does not require the collection over a large range of contrast and potentially different levels of salience.

This technique exploits a basic property of smoothed stimuli whereby a stimulus that has a peak contrast twice the clock's threshold engages the clock for the same time independently of temporal smoothing. This invariance point is flanked by two regions with opposite behaviors. At lower contrasts (Figure 4A), the stimulus with more temporal smoothing (orange) engages the clock for very little and should be perceived as shorter. At higher contrasts (i.e., above two clock thresholds, Figure 4B) the stimulus with more smoothing engages the clock for more time and is perceived as longer. Indeed the transition from a region where more smoothing is more time to a region where more smoothing is less time indicates that the indifference point has been crossed. Interestingly the estimate remains valid even if a low pass perceptual stage adds onto the temporal smoothing embedded in the stimulus.

Figures 4C,D present the results for luminance modulated stimuli for two subjects. In both subjects a stimulus contrast of three visibility limens is below the invariance point of twice the clock' threshold and the stimuli with less temporal smoothing are those which are perceived as longer. However as soon as the contrast is increased to five or six visibility limens, the stimuli with higher temporal smoothing begin to be perceived as longer indicating that the threshold has been exceeded. Spline interpolation among the data points indicate that point where the two curves cross (i.e., the invariance point) amounts to 4.26 and 5.82 for the two subjects, indicating that clock threshold for achromatic stimuli is between 2 and 3 visibility thresholds.

The results for the color modulated stimuli are presented in Figures 4E,F. In this case the critical value corresponding to twice the clock's threshold is higher: 10.8 and 12.5 for subjects GMC and OS. This indicates that the clock's threshold for colored stimuli is comprised between five and six visibility thresholds, a much larger value than luminance stimuli threshold.

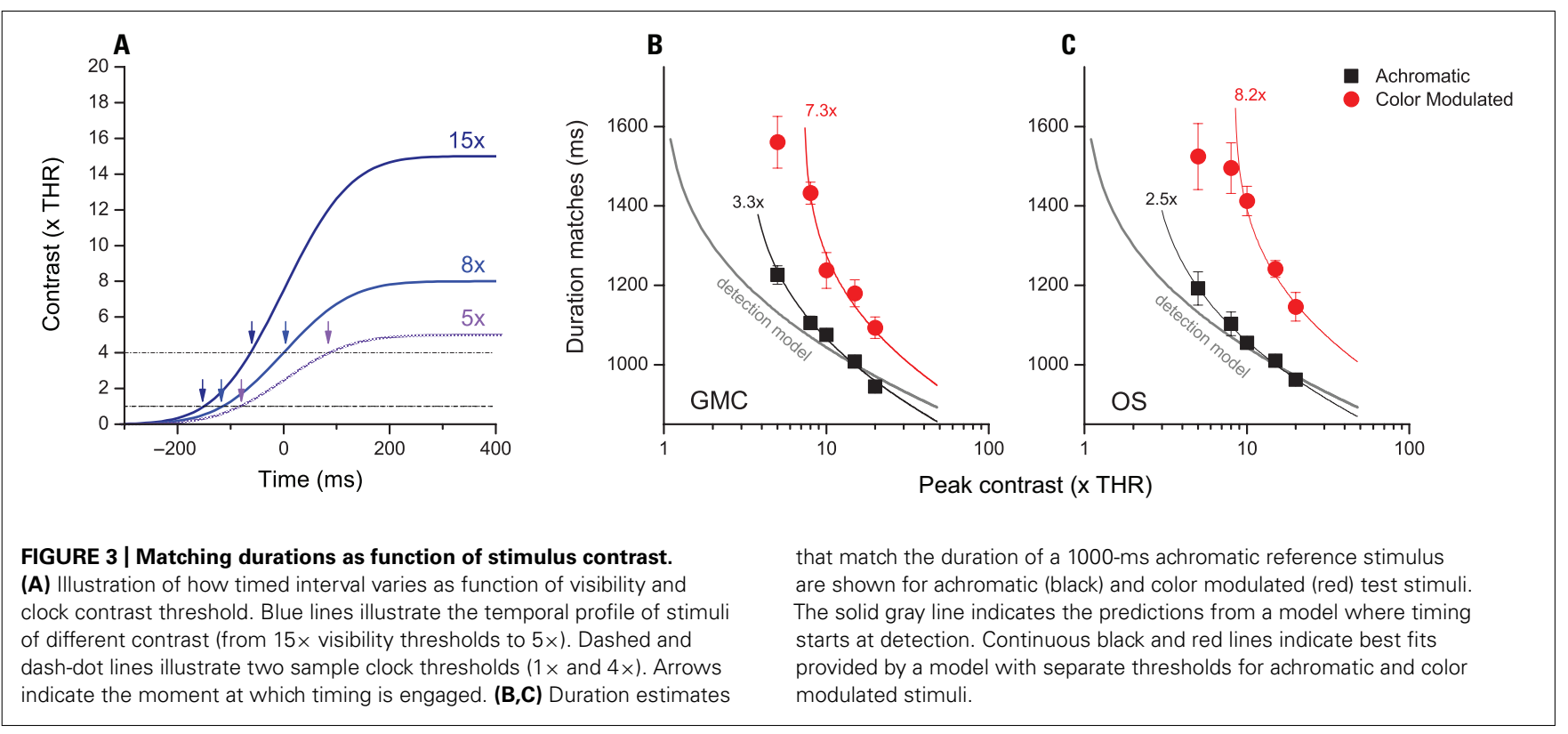




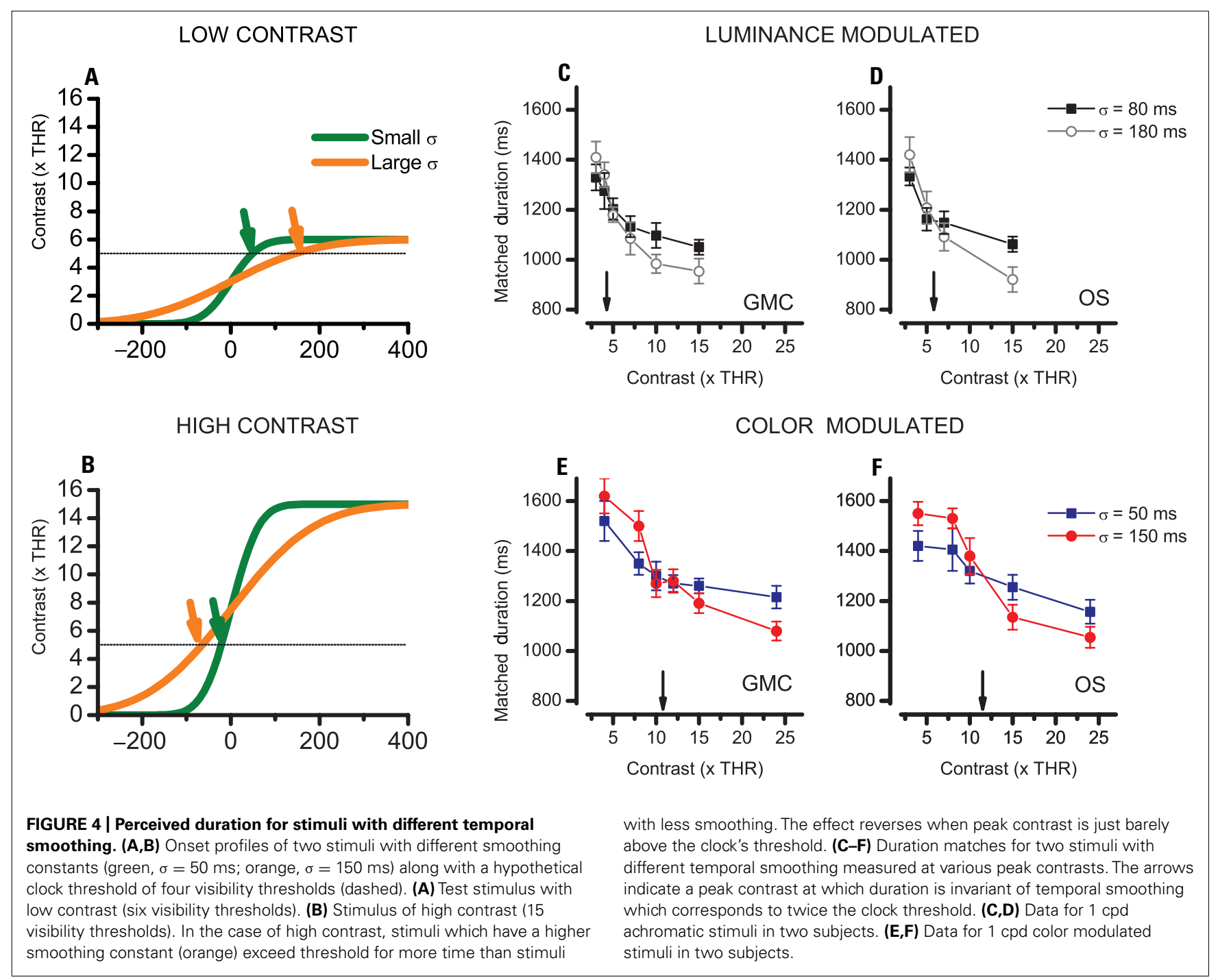

\section{Audiovisual temporal order judgment}

One last procedure to assess the properties of the mechanism which start and stop the integration of time has been a multimodal judgment where the subjects compare the timing of the onset or offset of the visual stimulus to a reference brief auditory tone. Figure 5A shows the average PSE for the Onset Audio Visual TOJ and Figure 5B shows the results for the Offset AVTOJ for two subjects. The data indicate that the onset of luminance modulated stimulus is timed much earlier than the onset of the color modulated stimulus. On the other hand the offset of the two stimuli yield similar estimated timings.

Although a direct comparison with duration matching experiments is not possible this result indicates that the difference in processing the temporal markers is in the same ballpark as the duration compression. On a face value the temporal distortion seems asymmetric and somewhat questions the idea of a common threshold for onset and offset detection. However, it must be considered that the color modulated stimuli are relayed via a channel that has sluggish dynamics and this may introduce a delay in the overall response. To illustrate the point I have simulated the response to a 1000-ms stimulus of two filters having the dynamics of the luminance and color pathways as inferred from the literature (Burr and Morrone, 1996; Figure 5C, continuous black and red lines). Interestingly, when we employ the thresholds inferred from previous procedures one obtains a pattern of results similar to the experimental data. The onset of colored stimuli is delayed both due to a sluggish response and to a higher clock threshold. At the offset the two factors work against each other and predict similar perceived offsets for luminance and colored stimuli (Figure 5C, arrows).

\section{DISCUSSION}

In this work I have explored the mechanisms that mediate the duration of stimuli relayed via the parvocellular pathway. In a first set of data I reported that color modulated stimuli last less than luminance modulated stimuli of equal visibility. The effect is quite large with an average difference of $185 \mathrm{~ms}$ in favor of luminance modulated stimuli. At odds with other forms of duration compression like those induced by attentional decrement or by adaptation (Johnston et al., 2006; Cicchini and Morrone, 2009) 


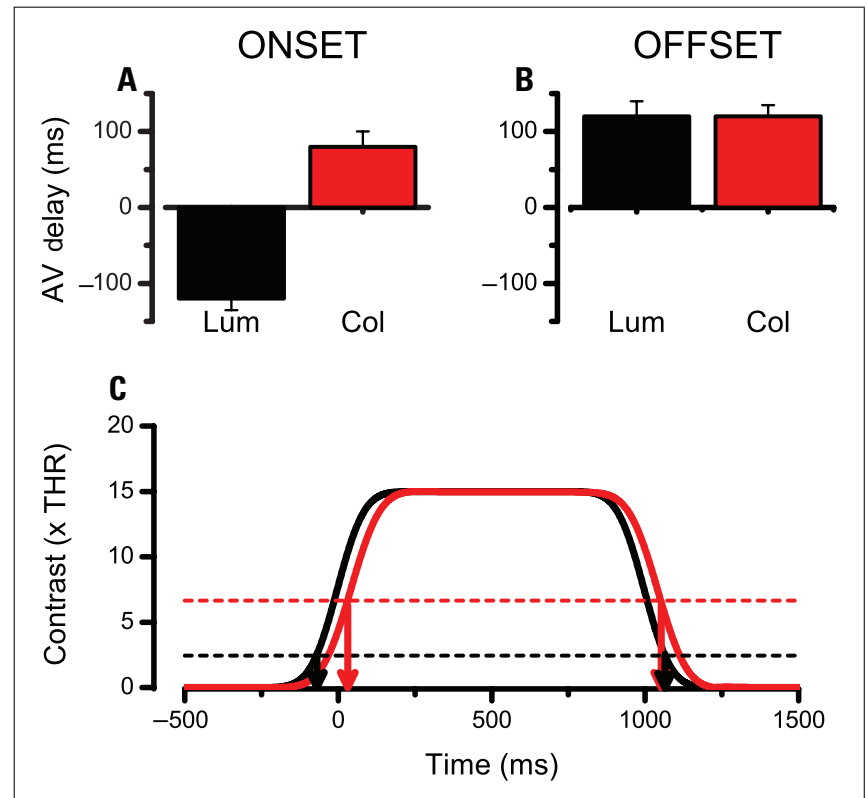

FIGURE 5 | Perceived onset and offset of the visual stimulus as measured with an AVTOJ. (A,B) Average perceived onset and offset of a luminance modulated stimulus (black) or a color modulated stimulus (red) for two subjects. Positive values indicate that a sound had to be presented after the visual event to be perceived as simultaneous. Hence positive values correspond to the visual event to be perceived after its nominal occurrence. (C) Illustration of how a higher threshold for color modulated stimuli combined with more sluggish relay of visual information predicts a great effect at the onset and a very small effect at the offset (see text for more details).

the bias is constant at all durations tested. This suggests that the difference arises more in the mechanisms that delimit the stimulus than those that integrate the passage of time.

The effect seems to be specific for color modulated stimuli and does not occur for other stimuli conveyed by the parvocellular system such as high spatial frequency luminance modulated gratings. This indicates that both the magnocellular and the parvocellular systems are able to produce reliable temporal estimates provided that they are engaged by suitable stimuli.

These conclusions presented here bear upon the assumption that $1 \mathrm{cpd}$ equiluminant gratings and $8 \mathrm{cpd}$ achromatic gratings are able to excite exclusively the parvocellular pathway. At present some controversy exists on the possibility to obtain such selective responses. In particular monkey recordings show that gratings of $8 \mathrm{cpd}$ drifting at $4 \mathrm{~Hz}$, even if not optimally, may still drive a subpopulation of 5-10\% of magnocellular neurons (Levitt et al., 2001; Alitto et al., 2011). One main difference between those studies and the present one is temporal frequency: in most neurophysiological recordings a temporal frequency of $4 \mathrm{~Hz}$ is employed, here the stimulus which is sustained and filtered by a temporal Gaussian has a spectrum well contained below $2 \mathrm{~Hz}$. Physiological data on such fine conditions are scarce, but some evidence indicates that at lower temporal frequencies, spatial cut-off frequencies of magnocellular neurons is lower with responses stopping at about 2-3 cpd (see Figure 13 in Derrington and Lennie, 1984). In addition studies in humans show that achromatic contrast sensitivity is mediated by magnocellular neurons only up to $2 \mathrm{cpd}$ and by parvocellular neurons from 4 cpd onward (Leonova et al., 2003). In any case, even considering the highest spatial cut-off frequencies, it is quite clear that $8 \mathrm{cpd}$ luminance gratings are much less optimal than $1 \mathrm{cpd}$ gratings in engaging the magnocellular system and if duration perception were mediated only by magnocellular neurons one should still have expected a large difference between 1 and $8 \mathrm{cpd}$.

One of the most prominent differences between magnocellular and parvocellular responses are their temporal impulse response functions (Morrone et al., 2005; Schutz et al., 2009). In particular, the parvocellular neurons have high temporal integration constants and operate as low pass temporal filters. However, simple simulations show that the stimulus which is filtered with longer constants (i.e., the parvocellular) is more smeared in time and thus it should be perceived as longer. This indicates that by itself temporal smoothing cannot explain the results reported here.

One simple explanation for the difference in perceived duration for the two types of stimuli is to postulate that the measurement of duration is engaged at different visibility levels for the two stimuli. In the paper the three techniques employed all suggest that timing of color modulated stimuli starts at higher visibility levels than timing of luminance modulated stimuli.

The first evidence is based on the dependence of perceived duration upon stimulus visibility. Data collected in a wide range of contrasts indicated that perceived duration decreases at lower contrasts consistent with the idea that timing starts only at a given visibility level. Best fitting curves for the $1 \mathrm{cpd}$ achromatic and the 1 cpd color modulated stimulus yield different clock thresholds, about two detection thresholds for the achromatic and six detection thresholds for the equiluminant stimulus. This model not only predicted well the experimental data (average $R^{2}=0.95$ ) but also fared better than a model assuming a constant bias between the two types of stimuli $\left(R^{2}=0.65\right)$.

A second technique exploited the property that when the contrast of the stimuli is twice that necessary for timing, the duration estimates are the same regardless of smoothing of the onset and of offset. This procedure yields reliable clock thresholds estimates which are independent of temporal smoothing (even those introduced by visual analysis), stimulus type and visual salience. Also in this case the threshold for color modulated events was estimated to be three times that for achromatic stimuli.

Finally I employed audiovisual temporal order judgment. These results indicated that the decrease of perceived duration of the color modulated stimuli can be ascribed to a mislocalization of the event markers. The difference is not symmetrical and resides mostly at the onset of visual stimuli. This is probably because at stimulus onset the sluggish response to colored stimuli adds up to the effect of a higher threshold.

Overall the simple hard threshold model has been quite successful in predicting perception of duration in a variety of tasks. Whereas in general perceived duration may depend on factors such as alertness, stimulus complexity and the like, these data indicate that the difference in duration of achromatic and chromatic stimuli depends almost entirely by way the onset and the offset of the stimulus are timed. 
Interestingly the effect reported here shares many similarities with reaction times to equiluminant stimuli (Burr et al., 1998; Burr and Corsale, 2001; McKeefry et al., 2003). Reaction times to equiluminant stimuli are about $200 \mathrm{~ms}$ slower than to luminance modulated stimuli of equal visibility, very similarly to what happens for the onset of visual stimuli. Burr and Corsale (2001) have proposed an explanation for these differences based on different gain mechanisms along the magnocellular and parvocellular pathways with the former saturating at lower contrast values and the latter proceeding more linearly. Indeed a difference in gain at low visibility levels is compatible with the idea that achromatic stimuli engage the clock at low visibility levels whilst equiluminant stimuli do not engage the clock up to higher contrasts.

\section{REFERENCES}

Alitto, H. J., Moore, B. D. T., Rathbun, D. L., and Usrey, W. M. (2011). A comparison of visual responses in the lateral geniculate nucleus of alert and anaesthetized macaque monkeys. $J$. Physiol. 589, 87-99.

Ayhan, I., Bruno, A., Nishida, S., and Johnston, A. (2009). The spatial tuning of adaptation-based time compression. J. Vis. 9, 2.1-2.12.

Ayhan, I., Bruno, A., Nishida, S., and Johnston, A. (2011). Effect of the luminance signal on adaptationbased time compression. J. Vis. 11, 22.

Binda, P., Cicchini, G. M., Burr, D. C., and Morrone, M. C. (2009). Spatiotemporal distortions of visual perception at the time of saccades. J. Neurosci. 29, 13147-13157.

Brown, S. (1995). Time, change, and motion: the effects of stimulus movement on temporal perception. Percept. Psychophys. 57, 105-116.

Buonomano, D. V., and Merzenich, M. M. (1995). Temporal information transformed into a spatial code by a neural network with realistic properties. Science 267, 1028-1030.

Burr, D., Silva, O., Cicchini, G. M., Banks, M. S., and Morrone, M. C. (2009). Temporal mechanisms of multimodal binding. Proc. Biol. Sci. 276, 1761-1769.

Burr, D., Tozzi, A., and Morrone, M. C. (2007). Neural mechanisms for timing visual events are spatially selective in real-world coordinates. Nat. Neurosci. 10, 423-425.

Burr, D. C., and Corsale, B. (2001). Dependency of reaction times to motion onset on luminance and chromatic contrast. Vis. Res. 41, 1039-1048.

Burr, D. C., Fiorentini, A., and Morrone, C. (1998). Reaction time to motion onset of luminance and chromatic gratings is determined by perceived speed. Vis. Res. 38, 3681-3690.

Burr, D. C., and Morrone, M. C. (1996). Temporal impulse response functions for luminance and colour during saccades. Vis. Res. 36, 2069-2078. L., Giusti, M., and Burr, D. C. (2012). Optimal encoding of interval timing in expert percussionists. J. Neurosci. 32, 1056-1060.

Cicchini, G. M., and Morrone, M. C. (2009). Shifts in spatial attention affect the perceived duration of events. J. Vis. 9, 9.1-9.13.

Cicchini, G. M., and Tomassini, A. (2008). Perception of duration in the parvocellular system. Perception 37,69 .

Derrington, A. M., and Lennie, P. (1984). Spatial and temporal contrast sensitivities of neurones in lateral geniculate nucleus of macaque. J. Physiol. 357, 219-240.

Efron, B., and Tibshirani, R. J. (1994). An Introduction to the Bootstrap. New York: Chapman \& Hall/CRC.

Fujisaki, W., Shimojo, S., Kashino, M., and Nishida, S. (2004). Recalibration of audiovisual simultaneity. Nat. Neurosci. 7, 773-778.

Gegenfurtner, K. R., and Kiper, D. C. (2003). Color vision. Annu. Rev.

Goodfellow, L. D. (1934). An empirical comparison of audition, vision, and touch in the discrimination of short intervals of time. Am. J. Psychol. 46, 243-258.

Grondin, S. (1993). Duration discrimination of empty and filled intervals marked by auditory and visual signals. Percept. Psychophys. 54, 383-394.

Ingling, C. R. Jr., and Martinez-Uriegas, E. (1983). The relationship between spectral sensitivity and spatial sensitivity for the primate r-g X-channel. Vis. Res. 23, 1495-1500.
Cicchini, G. M., Arrighi, R., Cecchetti, Neurosci. 26, 181-206.

The bulk of these results indicates that luminance modulated stimuli are more effective in engaging temporal mechanisms. This division does not correspond to the division of visual afferents into magnocellular and parvocellular pathways and is well modeled assuming different contrast thresholds for timing the two types of stimuli.

\section{ACKNOWLEDGMENTS}

This research was supported by the ERC grants FP6-MEMORY and FP7-STANIB. The author would like to thank Alice Tomassini and Ottavia Silva for help in data collection and Maria Concetta Morrone for helpful discussions on the experimental design. Special thanks go to David Murphy and Eckart Zimmermann for reading an early version of the manuscript.

Ivry, R. B., and Spencer, R. M. (2004). The neural representation of time. Curr. Opin. Neurobiol. 14, 225-232.

Johnston, A. (2010). "Modulation of time perception by visual adaptation," in Attention and Time, eds. K. Nobre and J. Coull (New York, NJ: Oxford University Press), 187-200.

Johnston, A., Arnold, D. H., and Nishida, S. (2006). Spatially localized distortions of event time. Curr. Biol. 16, 472-479.

Johnston, A., Bruno, A., Watanabe, J., Quansah, B., Patel, N., Dakin, S., and Nishida, S. (2008). Visually-based temporal distortion in dyslexia. Vis. Res. 48, 1852-1858.

Kanai, R., Paffen, C. L., Hogendoorn, H., and Verstraten, F. A. (2006). Time dilation in dynamic visual display. $J$. Vis. 6, 1421-1430.

Kaneko, S., and Murakami, I. (2009) Perceived duration of visual motion increases with speed. J. Vis. 9, 14.

Kaplan, E., and Benardete, E. (2001) The dynamics of primate retinal ganglion cells. Prog. Brain Res. 134, 17-34.

Karmarkar, U. R., and Buonomano, D. V. (2007). Timing in the absence of clocks: encoding time in neural network states. Neuron 53, 427-438.

Leonova, A., Pokorny, J., and Smith, V. C. (2003). Spatial frequency processing in inferred PCand MC-pathways. Vis. Res. 43, 2133-2139.

Levitt, J. B., Schumer, R. A., Sherman, S. M., Spear, P. D., and Movshon, J. A. (2001). Visual response properties of neurons in the LGN of normally reared and visually deprived macaque monkeys. J. Neurophysiol. 85, 2111-2129.

Livingstone, M., and Hubel, D. (1988). Segregation of form, color, movement, and depth: anatomy, physiology, and perception. Science 240, 740-749.

Mauk, M. D., and Buonomano, D. V. (2004). The neural basis of temporal processing. Annu. Rev. Neurosci. 27, 307-340.

McKeefry, D. J., Parry, N. R., and Murray, I. J. (2003). Simple reaction times in color space: the influence of chromaticity, contrast, and cone opponency. Invest. Ophthalmol. Vis. Sci. 44, 2267-2276.

Miyazaki, M., Yamamoto, S., Uchida, S., and Kitazawa, S. (2006). Bayesian calibration of simultaneity in tactile temporal order judgment. Nat. Neurosci. 9, 875-877.

Morrone, M. C., Cicchini, M., and Burr, D. C. (2010). Spatial maps for time and motion. Exp. Brain Res. 206, 121-128.

Morrone, M. C., Ross, J., and Burr, D. (2005). Saccadic eye movements cause compression of time as well as space. Nat. Neurosci. 8, 950-954.

Roelofs, C. O., and Zeeman, W. P. C. (1951). Influence of different sequences of optical stimuli on the estimation of duration of a given interval of time. Acta Psychol. 8, 89-128.

Schutz, A. C., Braun, D. I., and Gegenfurtner, K. R. (2009). Improved visual sensitivity during smooth pursuit eye movements: temporal and spatial characteristics. Vis. Neurosci. 26, 329-340.

Stetson, C., Cui, X., Montague, P. R., and Eagleman, D. M. (2006). Motorsensory recalibration leads to an illusory reversal of action and sensation. Neuron 51, 651-659.

Terao, M., Watanabe, J., Yagi, A., and Nishida, S. (2008). Reduction of stimulus visibility compresses apparent time intervals. Nat. Neurosci. 11, 541-542.

van Wassenhove, V., Buonomano, D. V., Shimojo, S., and Shams, L. (2008). Distortions of subjective 
time perception within and across senses. PLoS ONE 3, e1437. doi: 10.1371/journal.pone.0001437

Watson, A. B., and Pelli, D. G. (1983). QUEST: a Bayesian adaptive psychometric method. Percept. Psychophys. 33, 113-120.

Yamazaki, T., and Tanaka, S. (2005). Neural modeling of an internal clock. Neural Comput. 17, 1032-1058.
Yarrow, K., Haggard, P., Heal, R., Brown, P., and Rothwell, J. C. (2001). Illusory perceptions of space and time preserve cross-saccadic perceptual continuity. Nature 414, 302-305.

Conflict of Interest Statement: The author declares that the research was conducted in the absence of any commercial or financial relationships that could be construed as a potential conflict of interest.

Received: 29 June 2011; paper pending published: 18 July 2011; accepted: 14 March 2012; published online: 28 March 2012.

Citation: Cicchini GM (2012) Perception of duration in the parvocellular system. Front. Integr. Neurosci. 6:14. doi: 10.3389/fnint.2012.00014

Copyright (C) 2012 Cicchini. This is an open-access article distributed under the terms of the Creative Commons Attribution Non Commercial License, which permits non-commercial use, distribution, and reproduction in othe forums, provided the original authors and source are credited. 